\title{
Resilience Characterization for Multilayer Infrastructure Networks
}

\author{
Mehmet Baran Ulak* \\ Is with the Department of Civil Engineering, University of Twente, Enschede, NB7512, \\ The Netherlands.Email:m.b.ulak@utwente.nl \\ Lalitha Madhavi Konila Sriram \\ Is with the Department of Electrical and Computer Engineering, Worcester Polytechnic \\ Institute, Worcester, Massachusetts, 01609, USA. Email: Ikonilasriram@wpi.edu
}

\section{Ayberk Kocatepe}

Is with the Connetics Transportation Group, Coral Springs, Florida, 33065, USA. Email: ayberk.kocatepe@gmail.com

\section{Eren Erman Ozguven}

Is with the Department of Civil and Environmental Engineering, Florida State University, Florida, 32310, USA. Email: eozguven@fsu.edu

\section{Reza Arghandeh}

Is with the Department of Computing, Mathematics and Physics, Western Norway University of Applied Sciences, Bergen, 5020, Norway.Email: arghandehr@gmail.com

\begin{abstract}
Catastrophic weather has significantly battered the U.S. Gulf Coast in recent years and exposed critical deficiencies in the resilience across communities and organizations. These deficiencies compel the devising of strategies to identify critical infrastructure components that require more attention with regard to building resilience. This article presents a holistic approach to assessing urban resilience by studying the coresilience of infrastructure networks. For this purpose, Tallahassee, Florida is used as a case study with a focus on both power and roadway networks and includes real-life disaster data from three extreme weather events that recently hit the study area. This article contributes to the coresilience concept through: 1) developing a geographical information system-based information-gathering approach to obtain an integrated infrastructure network and feed the causality models, 2) developing novel coresilience metrics to spatially identify and evaluate the high-risk locations, and 3) presenting a comprehensive case study and application of the developed approaches by using real-life data from three major storms that hit the study area.
\end{abstract}


C atastrophic weather has significantly battered the U.S. Gulf Coast in recent years and exposed weaknesses in emergency preparedness and response. Examples include hurricanes Hermine (2016) and Michael (2018) as well as a major storm in 2017 that hit the Florida Gulf Coast. These extreme weather events revealed critical deficiencies in coordination among communities, organizations, and governments. Emergency relief operations were significantly affected by these hurricanes, which left the impacted region with partially flooded roadways and downed trees, utility poles, and power lines. Moreover, the lack of coordination among entities slowed the restoration of the failed infrastructure components. For example, power-restoration crews had to slow down due to the roadway closures caused by debris (e.g., fallen trees)especially when the operations of the public-works crews, who are responsible for removing the debris, were not coordinated with restoration crews. Issues in coordination disproportionately impacted the populations at risk (e.g., lower income groups), who need and benefit from these services the most. Unfortunately, the magnitude and adverse outcomes of extreme events are expected to increase in the near future due to the effect of climate change. Furthermore, an aging infrastructure and increasing population are also contributing to the devastating effects of these extreme events.

The magnitude of the outcomes of such extreme events is predisposed by the ability of the infrastructure components to cope with the random and dynamic changes. In that context, infrastructure resilience can be defined as an infrastructure's ability to cope with challenges imposed by extreme events. Any shortfall in the infrastructure resilience can translate into deficiencies that can amplify the vulnerabilities of communities. Community resilience is a multidimensional characteristic defined by the layers of interconnected infrastructure networks. Therefore, the study of resilience should not only focus on a single network attribute but also study the interdependencies among multilayer infrastructure networks. In accordance with this, a concept named coresilience was previously developed by the authors of this article [1]. Within this coresilience concept, there is a need to 1) develop a geographical information systems (GIS)-based information-gathering approach to obtain an integrated infrastructure network and feed the causality models and 2) develop novel coresilience metrics to spatially identify and evaluate high-risk locations. These metrics can be used to develop multivariate prioritized-risk maps to assess community vulnerability at multiple scales and dimensions, including the impact of the storms on infrastructure, the environment, and communities. Moreover, a deep neural network causality-based approach (DNNC) is developed to identify the causality structure behind the resilience of infrastructure segments.
Developing mathematical metrics through the performance estimation of multiple infrastructure networks is a difficult task since infrastructure performance will definitely be affected by disaster-related disruptions, such as roadway closures due to flooding, storm surges, and fallen trees and utility poles. Therefore, a thorough assessment of the transportation and power networks, with a focus on integrating critical facilities, such as hospitals, land-use features, and storm-related factors, is particularly important to making a hierarchical prioritization possible. To understand and create an accurate hierarchical prioritization, it is necessary to comprehend the characteristic behavior of each infrastructure network and layer and the interdependency among these various networks.

It is important to note that modeling and analysis become challenging while dealing with the integration of various infrastructure networks due to reasons such as the curse of dimensionality, noise, irrelevant data, sparsity, and so on. The causality approach was utilized in this article to efficiently combine different networks. Although there have been several studies focusing on the identification of underlying causal relationships, there have been very few studies that leverage neural network algorithms for this purpose.

\section{Background}

It is crucial to investigate the structural properties of networks to understand the complex dynamics of these interdependent systems. In "Emergence of scaling in random networks" [2], the concept of scale-free networks and the Barabási-Albert model were introduced with their possible applications on natural, technological, and social systems. This was followed by an article [3] in which a method was developed to predict the growth dynamics of the individual vertices for the networks. For more information on the advancements with respect to complex networks, please refer to "Statistical mechanics of complex networks" [4]. Mathematical metrics were also developed to estimate the reliability of the networks. For example, the concept of network efficiency was introduced in "Efficient behavior of smallworld networks" [5] and refined in "Is the Boston subway a small-world network?" [6], with insights on the usage of this concept on real-world transportation networks. Several other models were proposed to evaluate the global and local efficiency properties of complex transportation networks [7], [8]. Results indicated that the failure of a single node is sufficient to affect the reliability of the entire network if the node is among the ones with the largest load.

Using the Barabási-Albert model, power networks were analyzed in Chassin et al. [9], whereas the cascade of failures and multiple dependent, competing failure processes were studied in Buldyrev et al. [10] and Peng et al. [11], respectively. The vulnerability of transportation networks has also been widely studied in the last two decades. Please 
refer to Berdica et al. [12] for more details. The word vulnerability was introduced by Sarewitz et al. [13] as a measure to describe the inherent characteristics of a system that create the potential for harm.

In Jenelius et al. [14], the concepts of link importance and site exposure were introduced to assess vulnerability and reliability through the increased travel cost due to roadway closures. An accessibility index was also derived to incorporate the distance-decay effect and the volume of traffic's influence on the transportation networks [15]. With a focus on the effect of disasters on traffic conditions, Sumalee et al. [16] developed an optimization model, using MonteCarlo simulation, to approximate the capacity reliability of a transportation network. Similar disaster-related studies, with a prioritization of the minimization of the disruption caused by random and targeted attacks on the networks, were conducted [17]. Other studies, with a concentration on the effect of disasters on roadway links, were also conducted [18]. An interesting study was presented by Jenelius [19], where long-term vulnerability issues were found to be directly related to the relationship between transportation networks and population densities. Recently, some studies aimed at causal characterizations of transportation, using the principles of causality. Causality theory is used in the literature to investigate the underlying relationships among different variables. There are many ongoing researches that focus on establishing better causality algorithms [20]. With the increasing popularity of neural networks, the utilization of neural-network algorithms to reveal underlying direct and indirect causal relationships also increased [21], [22]. In this article, we propose using deep neural networks (DNNs) to identify causal relationships under extreme weather conditions, aiming to evaluate the most informative dependent variables for vulnerability assessment and resilience characterization.

There are several studies that focus on the resilience of interdependent infrastructure systems [23]-[31]. For instance, Mao et al. [26] demonstrated that resilience estimations drop by approximately $20 \%$ (when interdependencies are taken into account) by using a power and water infrastructure. Similarly, Almoghathawi et al. [25] also prioritized the interdependent resilience of power and water networks and used an optimization approach to minimize the recovery period. The joint resilience of gas and power networks was studied by Ouyang and Wang [23]. However, the authors adopted one-way dependency (i.e., power influenced the gas network) between networks rather than interdependency and concentrated on system-wide restoration efficiency. Among these studies, Fotouhi et al. [24] prioritized quantifying the resilience of traffic and electric system from a system-wide performance perspective. However, the authors did not consider the resilience quantification of the individual components of the networks. These infrastructures exist for the communities relying on them, and, hence, resilience deficiencies in the infrastructure systems impact these communities [31], [32]. Thus, a population-vulnerability-based optimization model was proposed by Berfin et al. [31], which integrates the community perspective into resilience, unlike previous studies, to achieve a community resilience. Similarly, the vulnerability index in our study takes population into account in addition to the critical facilities. Among the resilience studies, a number focused on developing resilience metrics [33]-[36]. While some ([33], [36], and [34]) developed system-wide metrics to measure the infrastructure resilience, Kocatepe et al. [35] attempted to develop component-based metrics that can pinpoint the less-resilient parts of the whole network. A comprehensive review of resilience studies concentrating on interdependent infrastructure systems from theory and practice perspectives can be found in "Resilience engineering: theory and practice in interdependent infrastructure systems" [22].

Despite the availability of literature on the resilience of interdependent infrastructure systems, not many of these studies focus on the characterization of the interdependencies between transportation and electricity networks [24], [37]-[39] or evaluate the resilience and vulnerability of these networks [2], [10], [16], [18], [19], [40]. However, the impact of the hurricanes and the identification of strategies to avoid the possible disruptions should clearly be studied extensively [41]-[46] to develop an integrated approach that can consider the coresilience of these networks. Despite recent efforts [1], [35], there is still a lack of a holistic approach to assessing the infrastructure resilience through studying the coresilience of power and transportation networks together.

\section{Methodology}

The coresilience concept was developed by the authors of this study to quantify the integrated power-and-transportation network resilience [1]. In this article, previous work is improved by the development of a GIS-based, complete integration of power and roadway networks. As such, this article creates an infrastructure network that combines individual networks and contains all of the relevant information for them to fully assess the resilience of the infrastructure system against storms and hurricanes. Furthermore, the proposed methodology was utilized to assess the vulnerability and resilience of the infrastructure networks, with a focus on critical facilities, such as hospitals.

\section{The Development of Coresilience Metrics}

A detailed methodology was developed in this article to evaluate the impact of the storms on the infrastructure networks of the City of Tallahassee, Florida. For this purpose, a single infrastructure network was produced by merging roadway and power networks (Figure 1). 


\section{Vulnerability Index}

The vulnerability index was calculated by using (1). The squared values used in the metric impose a higher weight to the repeating failures in the same segment. Moreover, the weights of outages and closures on the same segment are squared separately to avoid an overestimation of the effect of failures that occurred due to the same event (e.g., a closure and an outage occurring during Hermine in one single segment), considering that roadway closures and outages are highly associated.

$$
\begin{gathered}
V_{i}=\left(\sum_{e=1}^{E} \boldsymbol{I}_{o_{e, i}}\right)^{2}+\left(\sum_{e=1}^{E} \boldsymbol{I}_{C_{e, i}}\right)^{2} \\
\text { for } \boldsymbol{I}=f(x)=\left\{\begin{array}{ll}
1, & \text { there is a closure/outage } \\
0, & \text { no closure/outage }
\end{array},\right.
\end{gathered}
$$

where $V_{i}=$ the vulnerability index of infrastructure segment $i, e$ is event $(e=1,2, \ldots, E), E$ is the total number of events (i.e., disasters) used in assessment; $\boldsymbol{I}_{o_{c, i}}$ is an indicator of whether there is an outage at segment $i$ during event $e$; and $\boldsymbol{I}_{C_{e, i}}$ is an indicator of whether there is a closure at segment $i$ during event $e$. $I$ is equal to 1 if there is a closure or outage and is 0 otherwise.

\section{Priority Index}

The priority index (PI) is developed to prioritize infrastructure components based on the importance of the facilities or customers they serve (i.e., a component serving to a hospital has priority over another component serving to a residential unit). The index was calculated using several variables (Figure 2) (e.g., facilities, land use, number of customers) associated with the infrastructure segment (a full list is given in Table 1). The index equation is as follows:

$$
P_{i}=\sum_{f} \beta_{f} * N_{f, i}, \quad \forall i, f
$$

where $P_{i}=$ the PI of infrastructure segment $i, \beta_{f}$ is the coefficient utilized to weigh the effect of factor $f$ (e.g., hospital) on the $P_{i}$, and $N_{f, i}$ is the extent/magnitude of the factor $f$ [e.g., the number of beds at the hospital, number of students at school (1 if there is a supermarket, 0 otherwise)] in regards to segment $i$. This article uses the available priority benchmarks for the critical facilities given in Kocatepe et al. [35] for the prioritization (Table 1). Note that the higher the criticality of a facility is, the higher the priority is as well. Hospitals have a PI based on their number of beds, and individual hospital priorities were calculated by the multiplication of index with bed numbers. Similarly, schools' PIs were calculated based on the number of enrolled students. However, customers and land-use features, such as residential and commercial entities, were given different priorities.

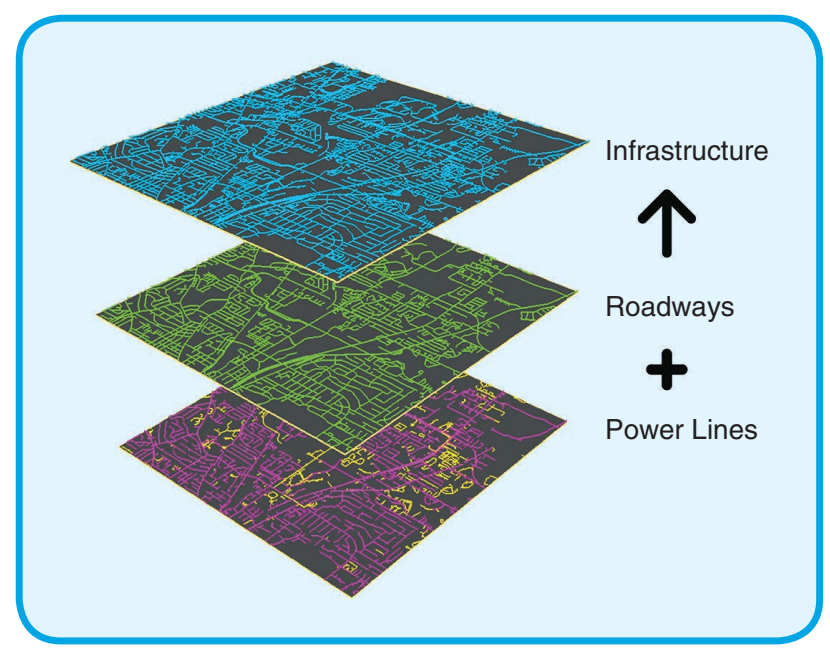

FIG 1 The integration of both roadway and power networks into one complete infrastructure network.

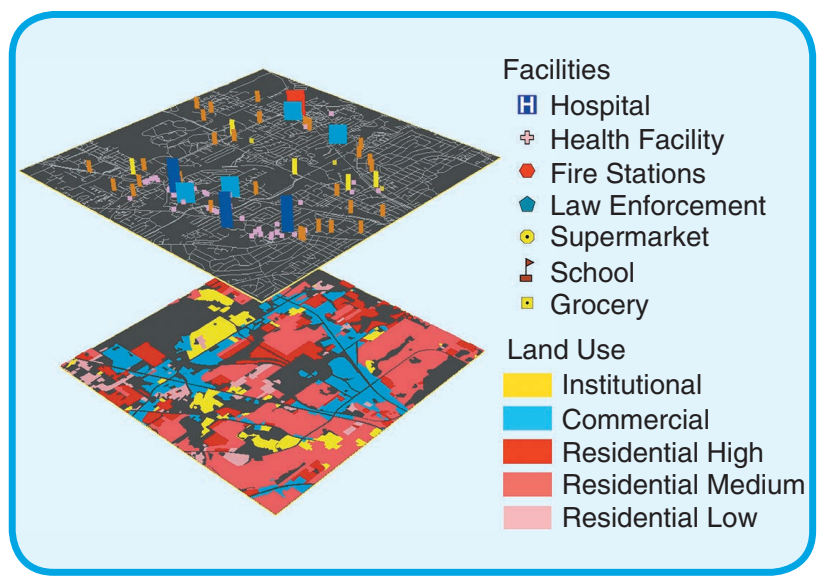

FIG 2 The facility and land-use features utilized to identify critical network components.

\section{Infrastructure Resilience Need}

Following the development of vulnerability and priority indices, the infrastructure-resilience need metrics $(R N)$ were calculated for each infrastructure segment $i$. These metrics can allow officials to spatiotemporally pinpoint segments that are preeminent while having the highest vulnerability to a hurricane. This metric is simply the normalized product of priority and feeder vulnerability (3):

$$
R N_{i}=\frac{V_{i} * P_{i}}{\max _{i}\left(V_{i} * P_{i}\right)}, \quad \forall i, \text { segment. }
$$

The PIs of facilities and the vulnerabilities of infrastructure segments are included within the $R N$ factor for every infrastructure segment.

\section{Causality Analysis}

To establish the proposed coresilience metrics, it is necessary to fathom the interconnectivity of these metrics under extreme weather conditions. Therefore, a causal mecha- 


\section{Table 1. The priority coefficients.}

$\begin{array}{lll}\text { Factor } & 2.51 \text { e-04 } & 0.193 \\ \text { Hospitals (per bed) } & 0.007 & - \\ \text { Health facility (Yes | No) } & 0.056 & - \\ \text { Fire stations (Yes | No) } & 0.030 & - \\ \text { Law enforcement (Yes | No) } & 0.016 & - \\ \text { Supermarket (Yes | No) } & 0.010 & - \\ \text { Grocery (Yes | No) } & 2.22 \mathrm{e}-05 & - \\ \text { School (per student) } & & - \\ \text { Land Use } & 0.050 & - \\ \text { Commercial (Yes | No) } & 0.050 & - \\ \text { Residential (high density) (Yes | No) } & 0.010 & - \\ \text { Residential (medium density) (Yes | No) } & 0.005 & - \\ \text { Residential (low density) (Yes | No) } & 0.050 & 0.05 \\ \text { Transportation (roadways) (Yes | No) } & 0.100 & - \\ \text { Utility buildings (Yes | No) } & 7.75 \mathrm{e}-07 & - \\ \text { Traffic volume (annual average daily traffic) } & 3.78 \mathrm{e}-05 & - \\ \text { Number of customers } & 6.25 \mathrm{e}-03 & - \\ \text { Feeder hierarchy (eight levels) } & & - \\ \end{array}$

nism was used to quantify the relatedness of the need for resilience to the effects of these extreme weather events. Causality analysis provides more intrinsic information on the interdependencies between the power and transportation networks while focusing on their vulnerability and on the aforementioned critical facilities.

Among other approaches to identifying and inferring causal relationships, a DNN-based approach has been utilized in this article due to its superiority [1]. Although there are several methods to identifying underlying causal relationships, based on the authors' previous studies, recurrent DNN was found to provide high accuracy and faster computation. This approach is based on recurrent neural networks, which can recognize more accurate patterns present in real-life, large data sets, such as power outages (POs) and roadway closures, and translate this information into vectors. DNNs, consisting of many hidden layers, are especially powerful in processing the input data. As such, this article utilized a neural network-based causality: the DNNC approach, developed in [1].

The selection of the optimal number of hidden layers is critical for the proposed methodology. This article uses a heuristic approach consisting of forward propagation for selecting the optimal number of hidden neurons. A layerwise implementation of the variance testing was applied to automatically determine the number of hidden units of hidden layers in the DNNC algorithm. In other words, various numbers of hidden layers were added one by one until the optimal accuracy versus speed was achieved. This number was increased gradually through the evaluation of the neural network performance. Based on this approach, six hidden layers were selected for the DNNC method to identify the causal relationships among the input variables. Alternative input variables were evaluated for their global Markov property-significance values. That is, for any stochastic process, the conditional distribution was evaluated to have dependency on both present and past states but not on the future states. If variables were known to have strong global Markov property, they were fed into the first recurrent neural network, and, if not, they were fed into the second recurrent neural network units. Based on the conditional variance test between each pair of variables, the highest variance variables were fed into the final network, which yielded the final causal model in the form of a directed acyclic graph. A brief description of the architecture used in the methodology is illustrated in Figure 3.

In addition, suppose $A, B, \ldots Z$ are different time-series data sets that represent POs, roadway closures, and weather and demographical information, which were filtered based on the conditional variance. The conditional variance shows the variance of a random variable, $X$, given the values of the other one or more variable(s), such as $Y$. Consider two distinct variables, $X$ and $Y$. The conditional variance is defined as follows:

$$
\operatorname{Var}(X \mid Y=y)=E\left[X^{2} \mid Y=y\right]-\mu_{X \mid Y}(y)^{2} .
$$

On one hand, the variables having high conditional variances were fed into the first set of hidden layers. On the other hand, the inputs to the second set of hidden layers had low conditional variance. Activation functions were used to initialize the neurons of the deep learning network after the input categorization (i.e., low and high conditional variance groups). The logistic sigmoid activation function, shown in (5), was utilized:

$$
f(x)=\frac{1}{1+e^{-x}} .
$$

In addition to the logistic sigmoid function, this article utilized another activation function, known as the hyperbolic function, shown next:

$$
f(x)=\frac{e^{x}-e^{-x}}{e^{x}+e^{-x}}
$$

Following this conditional variance training, the outputs were filtered and characterized, as displayed in Figure 3. The aforementioned activation functions were used for the weight computation in the DNNC algorithm. Each input variable was cross measured for conditional variance with other time-series variables. In other words, a pairwise conditional variance test was performed for 


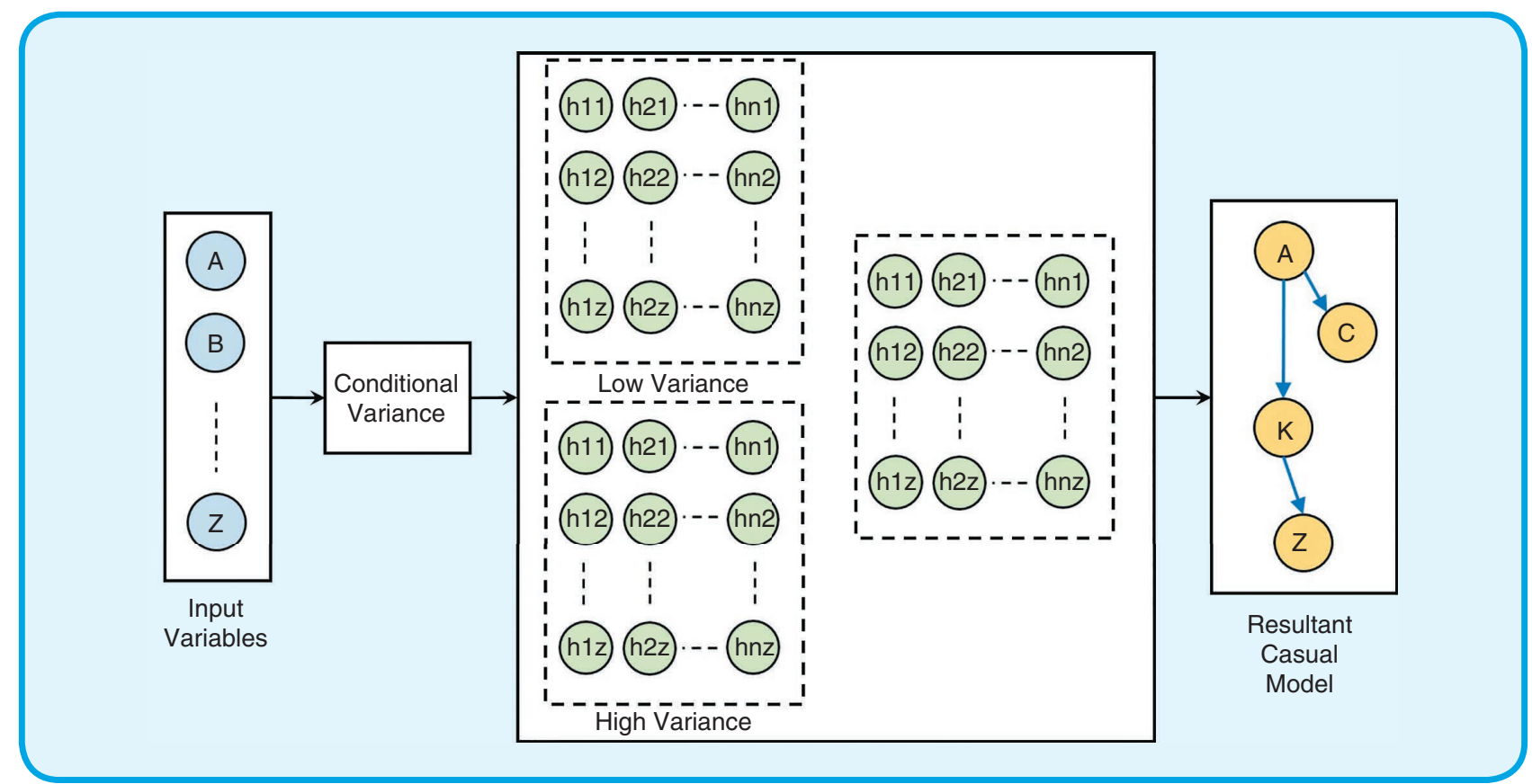

FIG 3 An overview of causality methodology.

all of the input variables. Variables were filtered based on the values obtained from the variance test. Based on the values of variance achieved, the variables were fed to the final DNN. The outputs from these two respective hidden layers formed causal graphs. These selected variables were fed into the final DNN output layer again. The advantage of utilizing a neural network approach is the efficient computation of the pairwise conditional variance values for all of the input variables. The outcomes of low-conditional-variance and high-conditional-variance DNNC units were fed into the final DNNC unit, leading to a causal graphical-model output in the context of the coresilience approach.

\section{Study Area and Data Description}

The City of Tallahassee, with a population of 286,272 [47], is the capital of Florida and a full-service municipality providing essential services to the region, including power, gas, water, solid waste, sewer, public works, an airport, and mass transit. Tallahassee has been hit by one storm and three major hurricanes since 2016. These extreme events, namely Hurricane Hermine (2016) [48], Hurricane Irma (2017) [49], Hurricane Michael (2018) [50], and the January storm (2016) [51], caused significant disruptions in the power grid and transportation network. The power and roadway infrastructures of Tallahassee were hit the hardest during the hurricanes Hermine and Michael. Hurricane Irma, on the other hand, did not directly hit Tallahassee. Rather, it had milder effects on the city, even though it was one of the most powerful hurricanes in recorded history.
The data resources used in this study were provided by the City of Tallahassee, including the POs and roadway closures as well as the whole power-and-roadwaynetwork infrastructure (Figure 4). Moreover, several critical facilities (e.g., five hospitals, 13 fire stations, and 14 police stations) are present in the city to serve the victims of disasters [Figure 5(a)]. The roadway and power infrastructure of the city is displayed in Figure 4(a) and Figure 4(b), respectively, whereas the merged infrastructure network is illustrated in Figure 4(c). Figure 5(a) presents the distribution of facilities in the city and land-use features of Tallahassee while Figure 5(b) outlines the land-use features (e.g., residential, commercial, and so on).

\section{Results, Discussion, and Validation}

\section{Vulnerability, Priority, and $R N$}

Developed metrics (see the histograms in Figure 6) helped illustrate the vulnerable and high-priority infrastructure components as well as the ones in need of improvement to enhance the resilience of the affected region (Figure 7). High-priority infrastructure segments identified via PI are displayed in Figure7(a) with bright pink colors. The impact of the critical facilities, such as hospitals and fire stations, on the PI can be observed by examining this figure together with Figure 5(a). Figure 7(b) provides the results for the vulnerability metric. High-priority locations, presented in Figure 7(a), are more clustered, and it is possible to detect these areas since PI is calculated using the area information, such as the land-use and facility locations. However, 


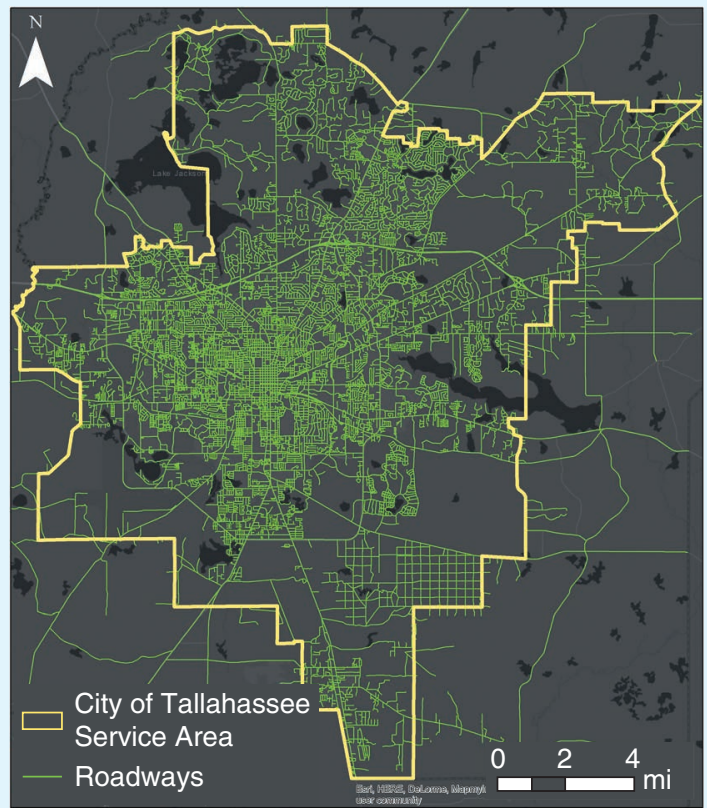

(a)

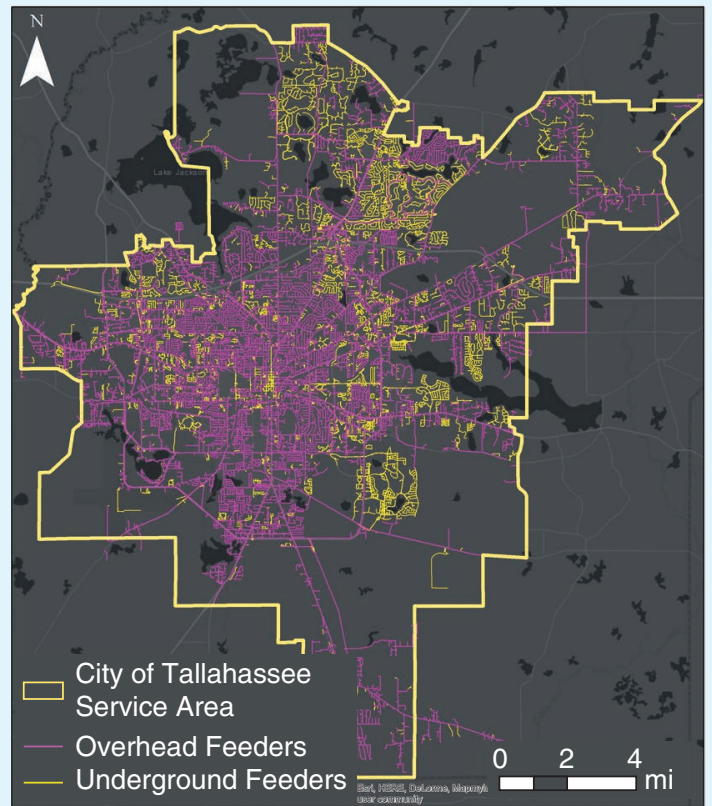

(b)

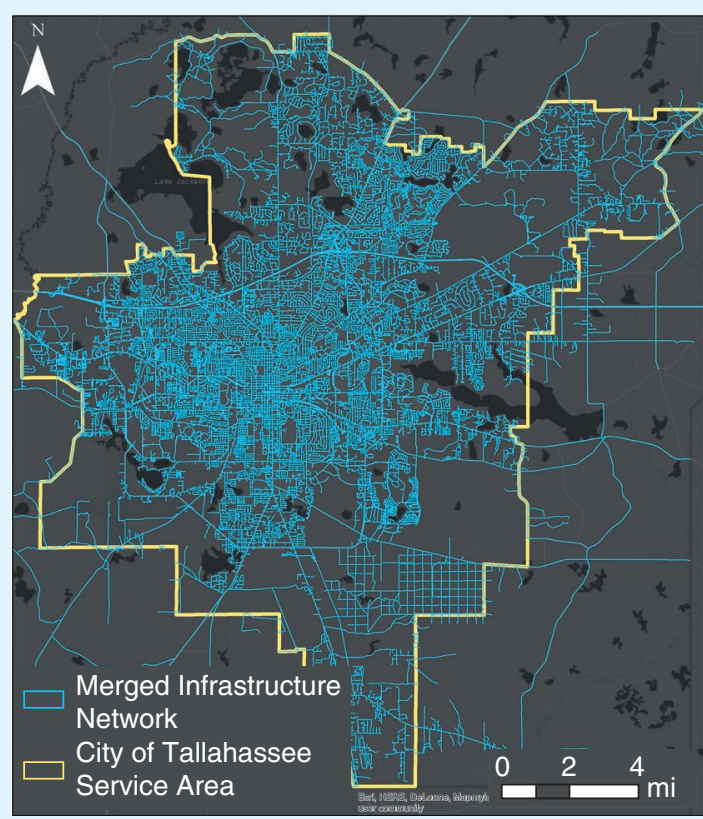

(c)

FIG 4 The study area's (a) roadway network, (b) power network, and (c) merged infrastructure network.

the vulnerability index is associated with individual links and the impacts of storms/hurricanes on those links. As such, highly vulnerable segments are dispersed rather than clustered, compared to the PI-based results.

The perfect solution would be to enhance the resilience of all infrastructure; however, it is generally not feasible, given the constrained budgets of the state and local governments that focus on emergency planning and response.
Therefore, it might be important for these agencies to allocate appropriate funds to increase the resilience of the most critical segments and locations. From this perspective, being vulnerable does not justify the need for resilience. However, it is required for a segment to be both high priority and vulnerable. The RN index provides such an assessment and identifies the most critical locations. Finally, RN illustrates those infrastructure segments, which the 


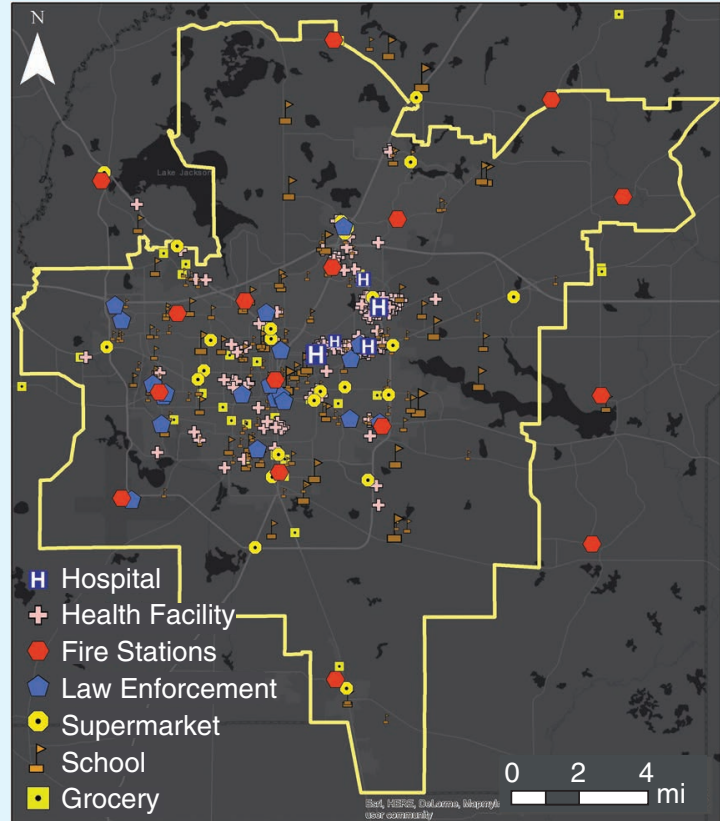

(a)

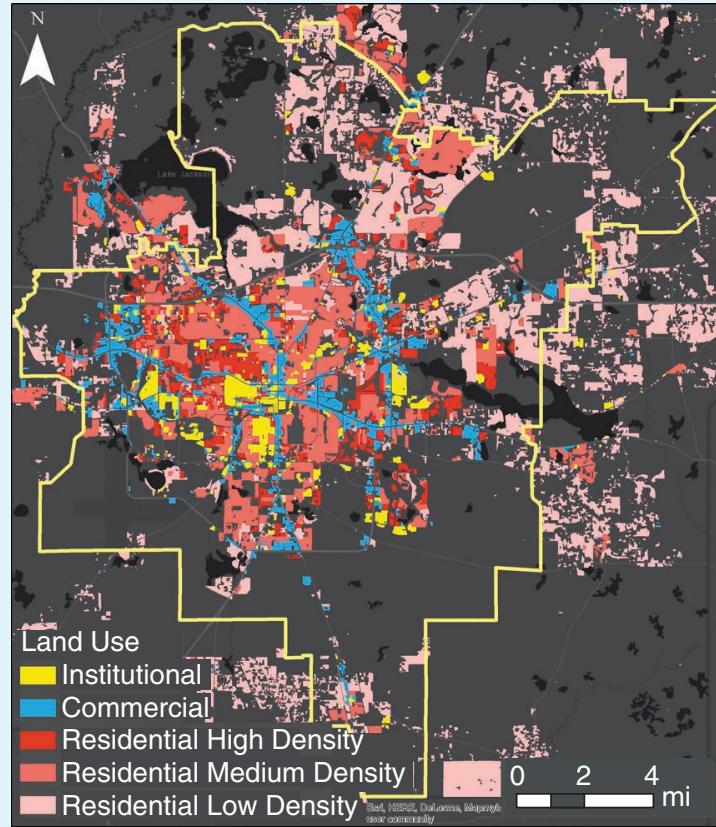

(b)

FIG 5 (a) The facilities in Tallahassee. (b) The land-use features.

city or any other relevant agency can identify and consider for future resilience-oriented investments.

\section{Cross-Validation with Causal Inference}

The DNNC algorithm was applied on the multidimensional data set consisting of weather variables, topographic variables, and infrastructure variables, including road closures (RCs) and failed power lines. The inputs to the DNNC network consist of time-series weather parameters, such as wind speed (WS), rain rate (RR), POs, roadway closures, and information regarding the critical infrastructure facilities and the topography. They also consist of information regarding the reasons for power failure, such as downed trees, affected power lines, downed transformers, and so on. The coresilience graphical models developed for Hurricane Hermine (2016), the January storm (2017), and Hurricane Michael (2018) are illustrated in Figure 8(a)-(c), respectively.

From Figure 8(a), it is observed that WS combined with RR causes a higher number of customers to be affected by POs. Moreover, fallen trees also give rise to RN. Although ground truth does state that weather variables, such as WS and RR, are primary causes of outages, information theory points to other variables, such as trees, power lines, and RCs, to be used to model RN. In comparison with Hurricane Hermine and Hurricane Michael, the tropical storm that occurred in January 2017 was much lower in intensity.

To validate the causal model on a lower-intensity event, DNNC was applied on the January storm data set as well. The resultant coresilience graphical model is illustrated in Figure 8(b). In this case, it is observed that the number of affected customers, POs, and roadway closures have causal-relationship RN, along with the downed power lines and trees and weather variables, as seen in Hurricane Hermine. This case is also a perfect example of the change of causal relationships with the change in the granularity of data and sparsity.

Similarly, Hurricane Michael also lists RC, the number of affected customers, and the power lines failed due to trees downed as causes of RN and lists WS and RR as the primary causes. With these causal diagrams, it becomes possible to precisely identify the most informative variables in modeling RN. Ground truth and common knowledge help us in identifying endogenous variables, but associated variables, common cause, and exogenous or hidden variables can be identified with the use of causality theory. Therefore, DNNC successfully identifies the underlying causal relationships across various infrastructure networks, which, in turn, can help in modeling a more resilient system.

\section{Conclusion and Future Work}

This article presented a holistic approach to assessing urban resilience by studying the coresilience of infrastructure networks. The infrastructure resilience of an urban region (Tallahassee) was studied using the data from three extreme weather events (one storm and two major hurricanes) that recently hit the Florida Gulf Coast in the United States. The resilience metrics proposed in this study aim to help 


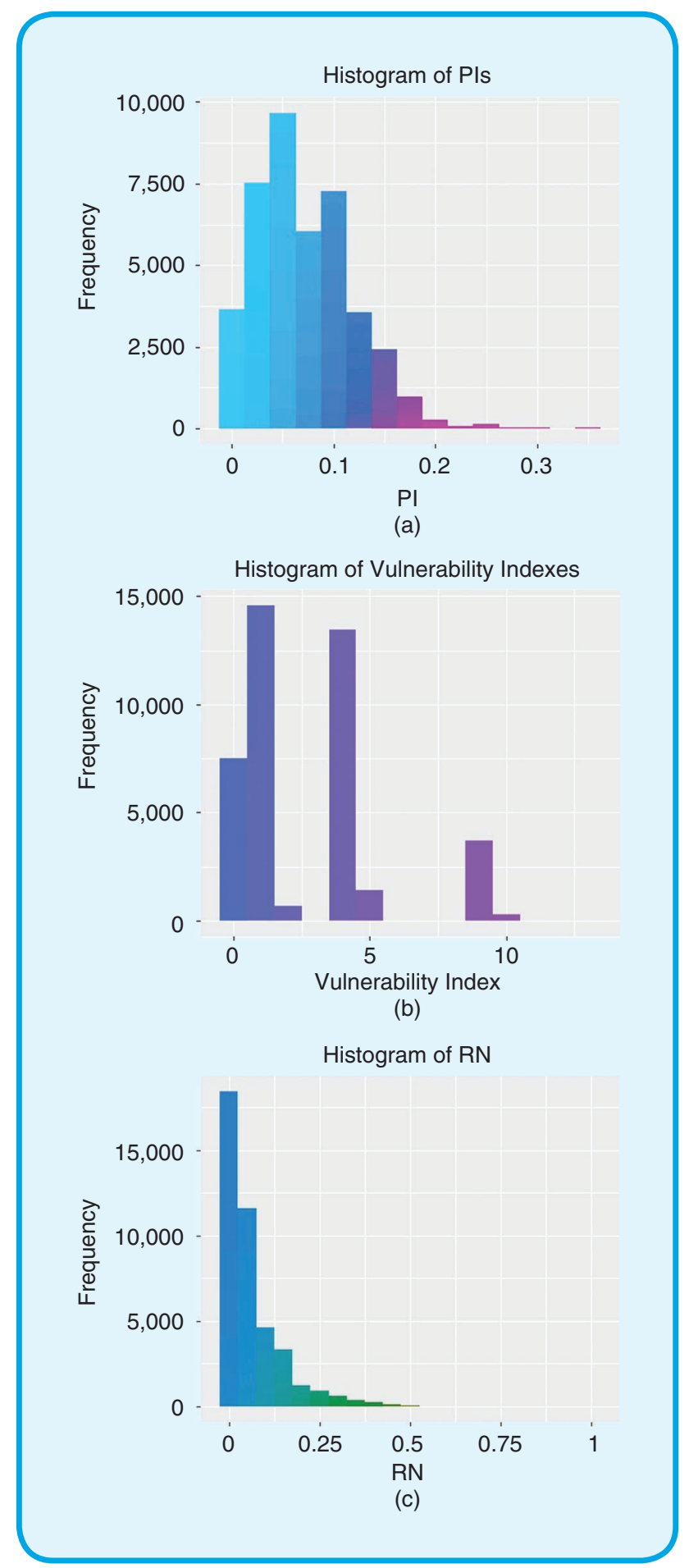

FIG 6 Histograms of (a) the PI, (b) vulnerability index, and (c) RN.

identify the most critical locations by incorporating both the priorities and vulnerabilities of infrastructure segments and aim to provide future resilience-oriented investments for these segments.

The resilience of transportation networks is not an isolated and independent problem and, hence, requires a coresilience-based assessment. Therefore, this study focuses on infrastructure resilience to assess transportation resilience. Such assessment can help other researchers to prioritize the interdependencies of roadway networks with other infrastructures, such as power and telecommunications networks. This is especially critical considering the future of transportation, with the widespread penetration of automated and connected vehicles as well as electric vehicles that will rely on functioning power and telecommunications infrastructures.

It is worth mentioning that there are certain limitations and caveats regarding the adopted methodology. The PI was calculated based on the spatial proximity of factors, such as facilities (e.g., hospitals, fire stations) or land-use features (e.g., commercial, residential), to the infrastructure segments. For example, an infrastructure segment closer to a fire station was considered as the one that could serve that station; hence, the PI of the segment was calculated accordingly. This is not a very strong assumption, considering that there is a high probability that spatial proximity is a valid approach to identifying the facilities, which are served by each infrastructure segment. However, there is still a need to enhance this accuracy. Even though such an identification task would require an extensive effort, this is a very promising future direction.

Another limitation is associated with the coefficients of the factors used in the calculation of the priority indices. Some of these coefficients were derived from previous research, such as that by Kocatepe et al. [35], and most of them were identified based on expert experience and knowledge. A better approach compels a better methodology to ensure that authorities and decision makers can identify more-accurate coefficients for the PI metric, which is also a very good future research opportunity.

\section{Acknowledgments}

This research is partly supported by U.S. NSF Award 1640587 . We would like to thank the City of Tallahassee, especially Michael Ohlsen, for providing the data and valuable insight. The contents of this article and discussion represent our opinion and do not reflect the official views of the National Science Foundation and the City of Tallahassee.

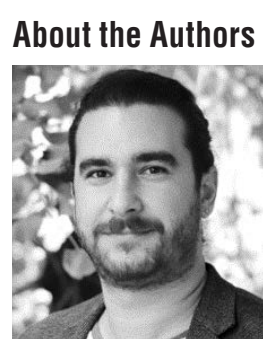

Mehmet Baran Ulak (m.b.ulak@ut wente.nl) earned his Ph.D. degree from the Department of Civil and Environmental Engineering, Florida State University in 2018. He is an assistant professor at the Department of Civil Engineering, University of Twente, Enschede, NB7512, The Netherlands. His research interests include transportation data analysis, resilience and disaster science, advanced statistical analysis, spatial analysis, and smart city concepts. 


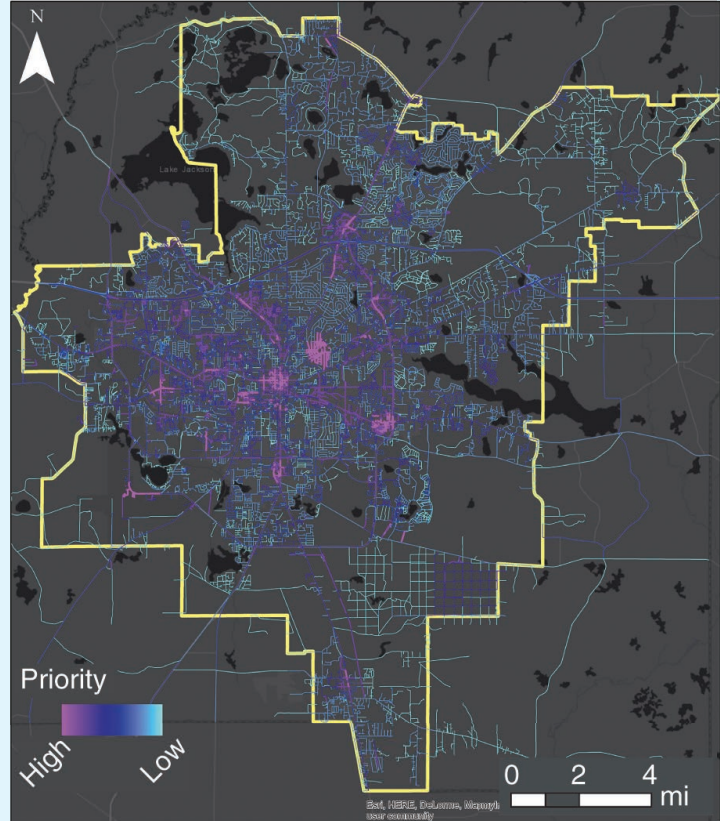

(a)

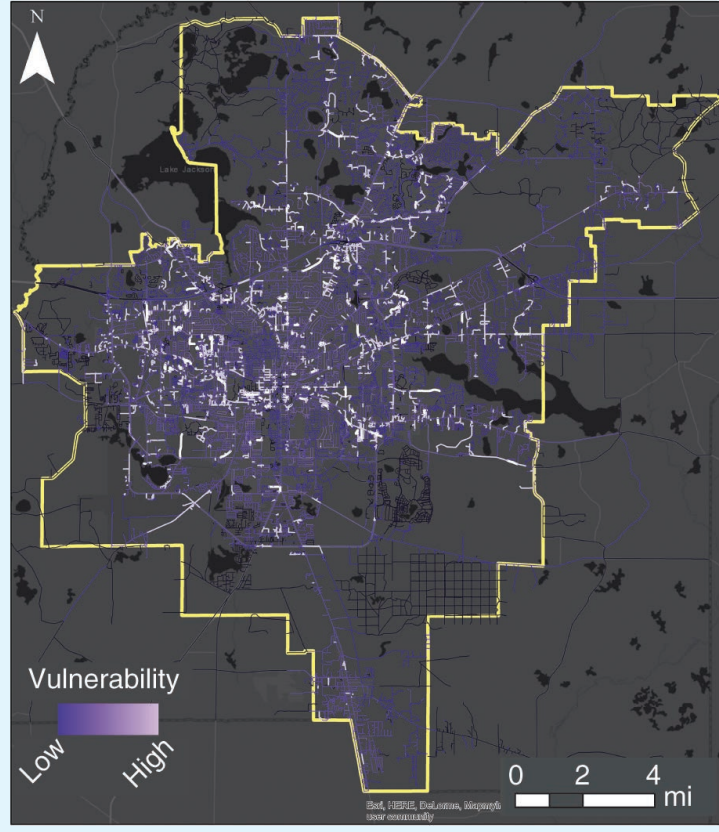

(b)

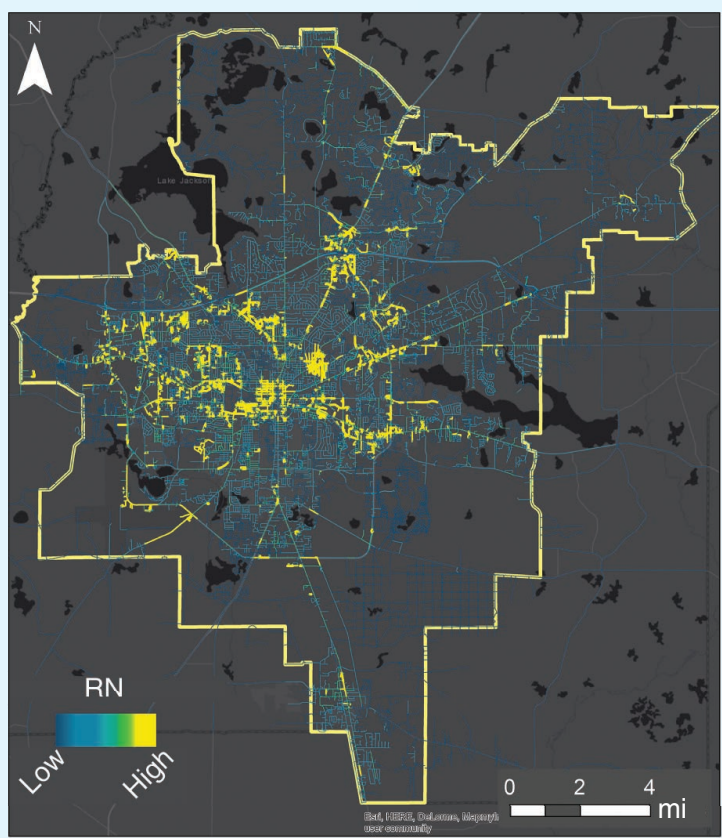

(c)

FIG 7 (a) The feeders priority, (b) feeders vulnerability, and (c) power RN.

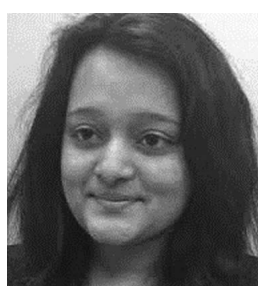

Lalitha Madhavi Konila Sriram (lko nilasriram@wpi.edu) earned her Ph.D. degree from the Department of Civil and Environmental Engineering, Florida State University in 2020. She is a postdoctoral researcher at the Worces- ter Polytechnic Institute, Worcester, Massachusetts, 01609, USA. Her research interests include the Internet of Things, machine learning, and data analysis for decision support in smart cities and grids. She is a Student Member of IEEE. 


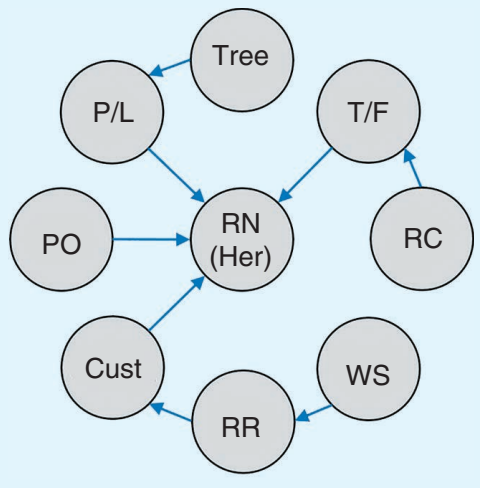

(a)

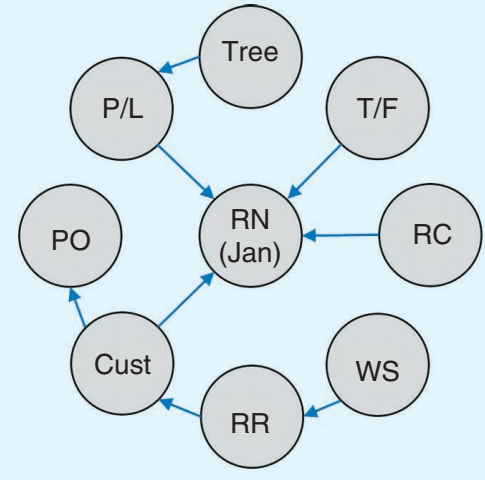

(b)

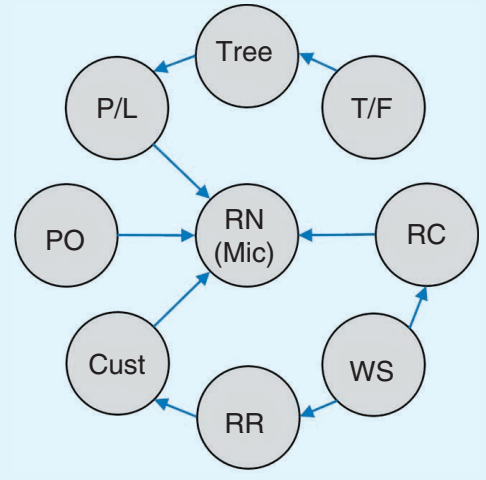

(c)

1) RN 2) P/L 3) T/F 4) WS 5) RR 6) PO 7) RC 8) Cust

FIG 8 The causality analysis result for: (a) Hurricane Hermine, (b) the January storm, and (c) Hurricane Michael. P/L: power line down; T/F: transformer down; Cust: number of customers.

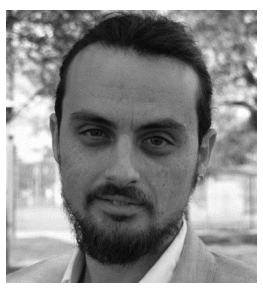

Ayberk Kocatepe (ayberk.kocatepe@ gmail.com) earned his Ph.D. degree from the Department of Civil and Environmental Engineering, Florida State University in 2017. He is a transportation modeler at the Connetics Transportation Group, Coral Springs, Florida, 33065, USA. His research interests include aging-focused emergency transportation operations, travel-time reliability, information dissemination via social networks, cloud computing and automated vehicles.

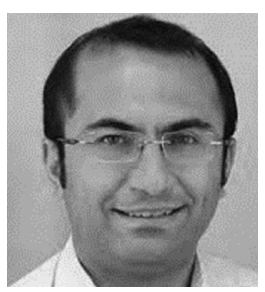

Eren Erman Ozguven (eozguven@fsu .edu) earned his Ph.D. degree in civil and environmental engineering from Rutgers University, with a focus on emergency supply transportation operations. He is an associate professor with the Department of Civil and Environmental Engineering, Florida A\&M University-Florida State University, Tallahassee, Florida, 32310, USA. His research interests include smart cities, urban mobility, transportation accessibility, traffic safety and reliability, emergency resilience, and intelligent transportation systems. He is a Member of IEEE.

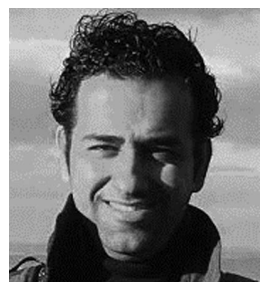

Reza Arghandeh (arghandehr@gmail .com) earned his Ph.D. degree in electrical engineering from Virginia Tech in 2013. He is a professor with the Department of Computing, Mathematics, and Physics and the director of the Collaborative Intelligent Infrastructure Lab, Western Norway University of Applied Sciences, Ber- gen, 5020, Norway. He is also a senior data scientist with StormGeo, Bergen, Norway. His research interests include machine learning, decision support for smart grids, causality analysis, and power system data analytics. He is a Senior Member of IEEE.

\section{References}

[1] K. S. Lalitha Madhavi, M. B. Ulak, E. E. Ozguven, and R. Arghandeh, "Multi-network vulnerability causal model for infrastructure co-resilience," IEEE Access, vol. 7, pp. 35344-35358, Mar. 11, 2019.

[2] A.-L. Barabasi and R. Albert, "Emergence of scaling in random networks," Science, vol. 286, no. 5439, pp. 509-512, Oct. 1999.

[3] A.-L. Barabási, R. Albert, and H. Jeong, "Mean-field theory for scalefree random networks," Physica A, Stat. Mech. Appl., vol. 272, nos. 1-2, pp. 173-187, 1999 .

[4] R. Albert and A.-L. Barabási, "Statistical mechanics of complex networks," Rev. Mod. Phys., vol. 74, no. 1, pp. 47-97, 2002. doi: 10.1103/ RevModPhys.74.47.

[5] V. Latora and M. Marchiori, "Efficient behavior of small-world networks,” Phys. Rev. Lett., vol. 87, no. 19, p. 198,701, 2001. doi: 10.1103/ PhysRevLett.87.198701.

[6] V. Latora and M. Marchiori, "Is the Boston subway a small-world network?" Phys. A Stat. Mech. Appl., vol. 314, nos. 1-4, pp. 109-113, Nov. 2002. doi: 10.1016/S0378-4371(02)01089-0.

[7] P. Crucitti, V. Latora, M. Marchiori, and A. Rapisarda, "Efficiency of scale-free networks: Error and attack tolerance," Phys. A Stat. Mech. Appl., vol. 320, pp. 622-642, Mar. 2003. doi: 10.1016/S03784371(02)01545-5.

[8] P. Crucitti, V. Latora, and M. Marchiori, "Model for cascading failures in complex networks," Phys. Rev. E. Stat. Nonlin. Soft Matter Phys., vol. 69, no. 4, p. 045104, 2004. doi: 10.1103/PhysRevE.69.045104.

[9] D. P. Chassin and C. Posse, "Evaluating North American electric grid reliability using the Barabási-Albert network model," Phys. A Stat. Mech. Appl., vol. 355, nos. 2-4, pp. 667-677, 2005. doi: 10.1016/j.physa.2005.02.051.

[10] S. V. Buldyrev, R. Parshani, G. Paul, H. E. Stanley, and S. Havlin, "Catastrophic cascade of failures in interdependent networks," Nature, vol. 464, no. 7291, pp. 1025-1028, Apr. 2010. doi: 10.1038/nature08932.

[11] H. Peng, O. Feng, and D. W. Coit, "Reliability and maintenance modeling for systems subject to multiple dependent competing failure processes," IIE Trans, vol. 43, no. 1, pp. 12-22, Oct. 2010. doi: 10.1080/0740817X.2010.491502.

[12] K. Berdica, "An introduction to road vulnerability: what has been done, is done and should be done," Transp. Policy, vol. 9, no. 2, pp. 117-127, Apr. 2002. doi: 10.1016/S0967-070X(02)00011-2.

[13] D. Sarewitz, R. Pielke, and M. Keykhah, "Vulnerability and risk: Some thoughts from a political and policy perspective," Risk Anal., vol. 23, no. 4, pp. 805-810, 2003. doi: 10.1111/1539-6924.00357. 
[14] E. Jenelius, T. Petersen, and L.-G. Mattsson, "Importance and exposure in road network vulnerability analysis," Transp. Res. A, Policy Pract. vol. 40, no. 7, pp. 537-560, Aug. 2006. doi: 10.1016/j.tra.2005.11.003.

[15] J. Sohn, "Evaluating the significance of highway network links under the flood damage: An accessibility approach," Transp. Res. A Policy Pract., vol. 40, no. 6, pp. 491-506, July 2006. doi: 10.1016/j.tra .2005.08.006

[16] A. Sumalee and F. Kurauchi, "Network capacity reliability analysis considering traffic regulation after a major disaster," Netw. Spat. Econ., vol. 6, nos. 3-4, pp. 205-219, Sept. 2006. doi: 10.1007/s11067-006-9280-0.

[17] Y. Lou and L. Zhang, "Defending transportation networks against random and targeted attacks," Transp. Res. Rec. J. Transp. Res. Board, vol. 2234, no. 1, pp. 31-40, Jan. 2011. doi: 10.3141/2234-04.

[18] E. Jenelius and L.-G. Mattsson, "Road network vulnerability analysis of area-covering disruptions: A grid-based approach with case study," Transp. Res. A, Policy Pract., vol. 46, no. 5, pp. 746-760, June 2012. doi: 10.1016/j.tra.2012.02.003.

[19] E. Jenelius, "Network structure and travel patterns: explaining the geographical disparities of road network vulnerability," J. Transp. Geogr., vol. 17, no. 3, pp. 234-244, May 2009. doi: 10.1016/j.jtrangeo.2008.06.002.

[20] Q. Ji, E. Bouri, R. Gupta, and D. Roubaud, "Network causality structures among Bitcoin and other financial assets: A directed acyclic graph approach," Q. Rev. Econ. Financ., vol. 70, pp. 203-213, Nov. 2018. doi: 10.1016/ j.qref.2018.05.016.

[21] A. Harell, S. Makonin, and I. V. Bajic, "Wavenilm: A causal neural network for power disaggregation from the complex power signal," in Proc. 44th Int. Conf. Acoustics, Speech, Signal Process. (ICASSP), 2019, pp. $8335-8339$.

[22] A. J. Hickford, S. P. Blainey, A. Ortega Hortelano, and R. Pant, "Resilience engineering: Theory and practice in interdependent infrastructure systems," Environ. Syst. Decis., vol. 38, no. 3, pp. 278-291, 2018. doi: 10.1007/s10669-018-9707-4.

[23] M. Ouyang and Z. Wang, "Resilience assessment of interdependent infrastructure systems: With a focus on joint restoration modeling an analysis," Reliab. Eng. Syst. Saf., vol. 141, pp. 74-82, Sept. 2015. doi 10.1016/j.ress.2015.03.011.

[24] H. Fotouhi, S. Moryadee, and E. Miller-Hooks, "Quantifying the resilience of an urban traffic-electric power coupled system," Re liab. Eng. Syst. Safety, vol. 163, pp. 79-94, July 2017. doi: 10.1016/ j.ress.2017.01.026.

[25] Y. Almoghathawi, K. Barker, and L. A. Albert, "Resilience-driven restoration model for interdependent infrastructure networks," Reliab. Eng. Syst. Saf., vol. 185, pp. 12-23, May 2019. doi: 10.1016/ j.ress.2018.12.006.

[26] Q. Mao and N Li, "Resilience assessment of interdependent critical infrastructure," in Proc. Joint Conf. Comput. Construction (JC3) (LC3 2017), 2017, vol. 1, pp. 121-128.

[27] R. Zimmerman, Q. Zhu, F. de Leon, and Z. Guo, "Conceptual modeling framework to integrate resilient and interdependent infrastructure in extreme weather," J. Infrastruct. Syst., vol. 23, no. 4, pp. 1-13, 2017. doi 10.1061/(ASCE)IS.1943-555X.0000394.

[28] J. Kong and S. P. Simonovic, "A model of interdependent infrastructure system resilience," Int. J. Safety Secur. Eng., vol. 8, no. 3, pp. 377-389, 2018. doi: 10.2495/SAFE-V8-N3-377-389.

[29] J. Kong, S. P. Simonovic, and C. Zhang, "Sequential hazards resilience of interdependent infrastructure system: A case study of greater Toronto area energy infrastructure system," Risk Anal., vol. 39, no. 5, pp. 1141-1168, 2019. doi: 10.1111/risa.13222.

[30] B. Rachunok and R. Nateghi, "Interdependent infrastructure system risk and resilience to natural hazards," in Proc. IISE Annu. Conf., 2019.

[31] D. Berfin, Y. Almoghathawi, K. Barker, and A. D. González, "International Journal of Disaster Risk Reduction Community resilience-driven restoration model for interdependent infrastructure networks," Int J. Disaster Risk Reduct., vol. 38, p. 101,228, Feb. 2019.

[32] M. B. Ulak, A. Yazici, E. E. Ozguven, A. Vanli, and R. Arghandeh, "Power resilience assessment from physical and socio-demographic perspectives," in Proc. 4th Int. Conf. Syst. Reliab. Saf. Power (ICSRS), 2019, pp. 421-428.

[33] R. Pant, K. Barker, and C. W. Zobel, "Static and dynamic metrics of economic resilience for interdependent infrastructure and industry sectors," Reliab. Eng. Syst. Saf., vol. 125, pp. 92-102, May 2014. doi: 10.1016/j.ress.2013.09.007.

[34] C. Nan, G. Sansavini, and, and W. Kröger, "Building an integrated metric for quantifying the resilience of interdependent infrastructure systems," in Critical Information Infrastructures Security. CRITIS 2014 (Lecture Notes in Computer Science, vol 8985), C. Panayiotou, G. Ellinas, E. Kyriakides, and M. Polycarpou, Eds. Berlin: Springer-Verlag, 2014, pp. 159-171.

[35] A. Kocatepe, M. B. Ulak, K. S. Lalitha Madhavi, D. Pinzan, E. E. Ozguven, and R. Arghandeh, "Co-resilience assessment of hurricane-induced power grid and roadway network disruptions: A case study in florida with a focus on critical facilities," in Proc. IEEE 21st Int. Conf. Intell. Transp. Syst. (ITSC), 2018, pp. 2759-2764.

[36] H. Baroud, K. Barker, J. E. Ramirez-Marquez, and C. M. Rocco, "Inherent costs and interdependent impacts of infrastructure network resilience," Risk Anal., vol. 35, no. 4, pp. 642-662, 2015. doi: 10.1111/risa.12223.

[37] K. S. Lalitha Madhavi et al., "Advanced electricity load forecasting combining electricity and transportation network," in Proc. 49th North American Power Symp., 2017, pp. 1-6.

[38] L. M. Konila Sriram, M. Gilanifar, Y. Zhou, E. Erman Ozguven, and R. Arghandeh, "Causal Markov Elman network for load forecasting in multinetwork systems," IEEE Trans. Ind. Electron., vol. 66, no. 2, pp. 1434-1442, Feb. 2019. doi: 10.1109/TIE.2018.2851977.

[39] S. Kelly, P. Tyler, and D. Crawford-Brown, "Exploring vulnerability and interdependency of UK infrastructure using key-linkages analysis," Netw. Spat. Econ., vol. 16, no. 3, pp. 865-892, 2016. doi: 10.1007/ s11067-015-9302-x.

[40] N. Goldbeck, P. Angeloudis, and W. Y. Ochieng, "Resilience assessment for interdependent urban infrastructure systems using dynamic network flow models," Reliab. Eng. Syst. Saf., vol. 188, pp. 62-79, Aug. 2019. doi: 10.1016/j.ress.2019.03.007.

[41] S. Sridhar, A. Hahn, and M. Govindarasu, "Cyber-physical system security for the electric power grid," Proc. IEEE, vol. 100, no. 1, pp. 210 224, Jan. 2012. doi: 10.1109/JPROC.2011.2165269.

[42] J. A. P. Lopes, C. L. Moreira, and A. G. Madureira, "Defining control strategies for microgrids islanded operation," IEEE Trans. Power Syst., vol. 21, no. 2, pp. 916-924, May 2006. doi: 10.1109/TPWRS 2006.873018 .

[43] D. Divan and H. Johal, "Distributed FACTS-A new concept for realizing grid power flow control," IEEE Trans. Power Electron., vol. 22, no. 6, pp. 2253-2260, Nov. 2007. doi: 10.1109/TPEL.2007.909252.

[44] R. Arghandeh et al., "The local team: Leveraging distributed resources to improve resilience," IEEE Power Energy Mag., vol. 12, no. 5, pp. 76-83, Sept. 2014. doi: 10.1109/MPE.2014.2331902.

[45] E. E. Ozguven et al., "Metadata-based needs assessment for emergency transportation operations with a focus on an aging population : A case study in Florida," Transp. Rev., vol. 36, no. 3, pp. 383412, 2016.

[46] E. E. Ozguven and K. Ozbay, "Emergency inventory management for disasters-A review," J. Emerg. Manag., vol. 12, no. 4, p. 269, Feb. 2016.

[47] "Households and families : 2010," U.S. Census Bureau, Washington, D.C., Rep. C2010BR-14, 2012. [Online]. Available: https://www. census.gov/content/dam/Census/library/publications/2012/dec/ c2010br-14.pdf

[48] R. Berg, "National Hurricane Center Tropical Cyclone Report - Hurricane Hermine," National Hurricane Center, National Oceanic and Atmospheric Administration, National Weather Service, Miami, FL, Rep. AL092016, 2016.

[49] J. P. Cangialosi, A. S. Latto, and R. Berg, "National Hurricane Center Tropical Cyclone Report-Hurricane Irma,” National Hurricane Center, National Oceanic and Atmospheric Administration, National Weather Service, Miami, FL, Rep. AL112017, 2018.

[50] "Hurricane Michael advisory archive," National Oceanic and Atmospheric Administration, Miami, FL, 2019. Accessed: Sept. 4, 2019. [Online]. Available: https://www.nhc.noaa.gov/archive/2018/ MICHAEL.shtml

[51] "Deadly Tornado outbreak," National Oceanic and Atmospheric Administration, 2017. Accessed: Sept. 4, 2019. [Online]. Available: https://www.weather.gov/tae/20170122_tornadoes 